\title{
The CuBA Library
}

\author{
T. Hahn \\ Max-Planck-Institut für Physik \\ Föhringer Ring 6, D-80805 Munich, Germany \\ Concepts and implementation of the CUBA library for multidimensional numerical integration are elucidated.
}

\section{Overview}

Cuba [1 is a library for multidimensional numerical integration. It features four integration algorithms with interfaces for Fortran, $\mathrm{C} / \mathrm{C}++$, and Mathematica. All four can integrate vector integrands and their invocation is quite similar to make it easy to switch routines for comparison. The main characteristics are summarized below.

Routine: Vegas

Basic integration methods available:

- Sobol sample (quasi Monte Carlo)

- Mersenne Twister sample (pseudo Monte Carlo)

Variance reduction: importance sampling

Routine: Suave

Basic integration methods available:

- Sobol sample (quasi Monte Carlo)

- Mersenne Twister sample (pseudo Monte Carlo)

Variance reduction: importance sampling combined with globally adaptive subdivision

Routine: Divonne

Basic integration methods available:

- Korobov sample (lattice method)

- Sobol sample (quasi Monte Carlo)

- Mersenne Twister sample (pseudo Monte Carlo)

- cubature rules (deterministic method)

Variance reduction: stratified sampling, aided by methods from numerical optimization

\section{Routine: Cuhre}

Basic integration method available:

- cubature rules (deterministic method)

Variance reduction: globally adaptive subdivision

Before explaining the buzzwords appearing in this list, it is perhaps worthwhile to address two frequently asked questions.
First, numerical integration rapidly becomes more difficult with increasing dimension, no matter how many tricks are built into the integrator. To illustrate this, imagine computing the volume of the $d$-dim. sphere $S_{d}$ by integrating its characteristic function $\chi=\theta\left(1-\|x\|_{2}\right)$ inside the surrounding hypercube $C_{d}=[-1,1]^{d}$. In a Monte Carlo way of thinking, then, the ratio $r=\operatorname{Vol} S_{d} / \operatorname{Vol} C_{d}$ can be taken as the chance that a general-purpose integrator will find the sphere at all. The numbers clearly display what is often termed the 'curse of dimensionality':

\begin{tabular}{c||c|c|c|c}
$d$ & 2 & 5 & 10 & 50 \\
\hline$r$ & .785 & .164 & .0025 & $1.5 \times 10^{-28}$
\end{tabular}

Second, CuBA (and, for that matter, most multidimensional integrators) can do only Riemann integrals of the form

$$
\mathbf{I} f:=\int_{0}^{1} \mathrm{~d}^{d} x f(\vec{x}) .
$$

Most questions concern the boundaries, although it is straightforward to transform a finite integration region to the unit hypercube:

$$
\begin{aligned}
& \int_{a_{1}}^{b_{1}} \cdots \int_{a_{d}}^{b_{d}} \mathrm{~d}^{d} x f(\vec{x})=\int_{0}^{1} \mathrm{~d}^{d} y J f(\vec{x}), \\
& J=\prod_{i=1}^{d}\left(b_{i}-a_{i}\right), \quad x_{i}=a_{i}+\left(b_{i}-a_{i}\right) y_{i} .
\end{aligned}
$$

\section{Concepts}

\subsection{Deterministic vs. Monte Carlo}

CuBA contains both deterministic and Monte Carlo integration methods. The deterministic ap- 
proach is based on cubature rules,

$$
\mathbf{I} f \approx \mathbf{C}_{n} f:=\sum_{i=1}^{n} w_{i} f\left(\vec{x}_{i}\right)
$$

with specially chosen nodes $\vec{x}_{i}$ and weights $w_{i}$. Error estimation is done e.g. by null rules $\mathbf{N}_{m}$ $(m<n)$ which are constructed to give zero for functions integrated exactly by $\mathbf{C}_{n}$ and thus measure errors due to "higher terms."

The Monte Carlo estimate, although quite similar in form,

$$
\mathbf{I} f \approx \mathbf{M}_{n} f:=\frac{1}{n} \sum_{i=1}^{n} f\left(\vec{x}_{i}\right),
$$

is conceptually very different as this formula denotes the statistical average over independent and identically distributed random samples $\vec{x}_{i}$. In this case the standard deviation furnishes a probabilistic estimate of the integration error:

$$
\sigma\left(\mathbf{M}_{n} f\right)=\sqrt{\mathbf{M}_{n} f^{2}-\mathbf{M}_{n}^{2} f} .
$$

\subsection{Construction of Cubature Rules}

Starting from an orthogonal basis of functions $\left\{b_{1}, \ldots, b_{m}\right\}$ - usually monomials - with which most $f$ can (hopefully) be approximated sufficiently well one imposes that each $b_{i}$ be integrated exactly by $\mathbf{C}_{n}: \mathbf{I} b_{i} \stackrel{!}{=} \mathbf{C}_{n} b_{i}$. These are $m$ moment equations

$$
\sum_{k=1}^{n} w_{k} b_{i}\left(\vec{x}_{k}\right)=\int_{0}^{1} \mathrm{~d}^{d} x b_{i}(\vec{x})
$$

for $n d+n$ unknowns $\vec{x}_{i}$ and $w_{i}$. They pose a formidable, in general nonlinear, system of equations. Additional assumptions, e.g. symmetries, are usually necessary to solve this system. CUBA employs the Genz-Malik rules [2] constructed from a symmetric monomial basis.

\subsection{Globally Adaptive Subdivision}

Once an error estimate for the integral is available, global adaptiveness is easy to implement:

1. Integrate the entire region: $I_{\mathrm{tot}} \pm E_{\mathrm{tot}}$.

2. while $E_{\text {tot }}>\max \left(\varepsilon_{\text {rel }} I_{\text {tot }}, \varepsilon_{\text {abs }}\right)$

3. Find the region $r$ with the largest error.
4. Bisect (or otherwise cut up) $r$.

5. Integrate each subregion of $r$ separately.

6. $I_{\mathrm{tot}}=\sum I_{i}, \quad E_{\mathrm{tot}}=\sqrt{\sum E_{i}^{2}}$.

7. end while

A remark is in order here about the two precisions, $\varepsilon_{\text {rel }}$ and $\varepsilon_{\text {abs }}$. Naively what one imposes is the relative precision: the result is supposed to be accurate to, say, one part in a thousand, i.e. $\varepsilon_{\text {rel }}=10^{-3}$. For integral values approaching zero, however, this goal becomes harder and harder to reach, and so as not to spend inordinate amounts of time in such cases, an absolute precision $\varepsilon_{\text {abs }}$ can be prescribed, where typically $\varepsilon_{\text {abs }} \ll \varepsilon_{\text {rel }}$.

\subsection{Importance Sampling}

Importance sampling introduces a weight function into the integral:

$$
\begin{aligned}
& \mathbf{I} f=\int_{0}^{1} \mathrm{~d}^{d} x w(\vec{x}) \frac{f(\vec{x})}{w(\vec{x})}, \\
& w(\vec{x})>0, \quad \mathbf{I} w=1,
\end{aligned}
$$

with two requirements: a) one must be able to sample from the distribution $w(\vec{x}), \mathrm{b}) f / w$ should be "smooth" in the sense that $\sigma_{w}(f / w)<\sigma(f)$, e.g. $w$ and $f$ should have the same peak structure. The ideal choice is known to be $w(\vec{x})=|f(\vec{x})| / \mathbf{I} f$ which has $\sigma_{w}(f / w)=0$, but is of little use as it requires a-priori knowledge of the integral value.

\subsection{Stratified Sampling}

Stratified sampling works by sampling subregions. Consider a total of $n$ points sampled in a region $r=r_{a}+r_{b}$ vs. $n / 2$ points sampled in $r_{a}$ and $n / 2$ in $r_{b}$. In the latter case the variance is

$$
\frac{1}{4}\left(\frac{\sigma_{a}^{2} f}{n / 2}+\frac{\sigma_{b}^{2} f}{n / 2}\right)=\frac{\sigma_{a}^{2} f+\sigma_{b}^{2} f}{2 n}
$$

whereas in the former case it can be written as

$$
\frac{\sigma^{2} f}{n}=\frac{\sigma_{a}^{2} f+\sigma_{b}^{2} f}{2 n}+\frac{\left(\mathbf{I}_{a} f-\mathbf{I}_{b} f\right)^{2}}{4 n} .
$$

Even in this simple example the latter variance is at best equal to the former one, and only if the integral values are identical. The optimal reduction of variance can be shown to occur for 
$n_{a} / n_{b}=\sigma_{a} f / \sigma_{b} f[3$. The recipe is thus to split up the integration region into parts with equal variance, and then sample all parts with same number of points.

\subsection{Quasi-Monte Carlo Methods}

Quasi-Monte Carlo methods are based on the Koksma-Hlawka inequality which states an upper bound on the error of an integration formula $\mathbf{Q}_{n} f=\frac{1}{n} \sum_{i=1}^{n} f\left(\vec{x}_{i}\right)$,

$$
\left|\mathbf{Q}_{n} f-\mathbf{I} f\right| \leqslant V(f) D^{*}\left(\vec{x}_{1}, \ldots, \vec{x}_{n}\right) .
$$

Apart from choosing a different integrand there is little one can do about $V(f)$, the "variation in the sense of Hardy and Krause." The discrepancy $D^{*}$ of a sequence $\vec{x}_{1}, \ldots, \vec{x}_{n}$ is defined as

$$
D^{*}=\sup _{r \in[0,1]^{d}}\left|\frac{\nu(r)}{n}-\operatorname{Vol} r\right|,
$$

where $\nu(r)$ counts the $\vec{x}_{i}$ that fall into $r$. The word "equidistributed" indeed commonly means that $\nu(r)$ is proportional to $\mathrm{Vol} r$. Quasi-random sequences can be constructed with a substantially lower discrepancy than (pseudo-)random numbers. A Monte Carlo algorithm based on these sequences typically achieves convergence rates of $\mathcal{O}\left(\log ^{d-1} n / n\right)$ rather than the usual $\mathcal{O}(1 / \sqrt{n})$.

CuBA offers a choice of quasi-random Sobol sequences [4] or pseudo-random Mersenne Twister sequences [5] for all Monte Carlo algorithms. Figure 1shows that quasi-random numbers cover the plane much more homogeneously than pseudorandom numbers.

\subsection{Lattice Methods}

Lattice methods require a periodic integrand, usually obtained by applying a periodizing transformation (CUBA's Divonne uses $x \rightarrow|2 x-1|$ ). Sampling is done on an integration lattice $L$ spanned by a carefully selected integer vector $\vec{z}$ :

$$
\begin{aligned}
& \mathbf{L}_{n} f=\frac{1}{n} \sum_{i=0}^{n-1} f\left(\left\{\frac{i}{n} \vec{z}\right\}\right), \\
& \{x\}=\text { fractional part of } x .
\end{aligned}
$$

$\vec{z}$ is chosen (by extensive computer searches) to knock out as many low-order "Bragg reflections"
Sobol Quasi-Random Numbers
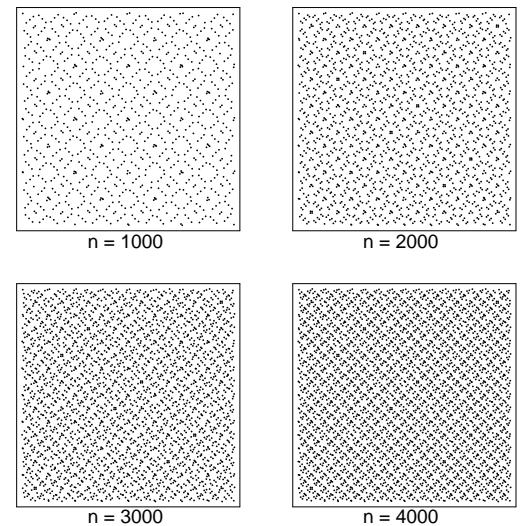

Mersenne Twister Pseudo-Random Numbers
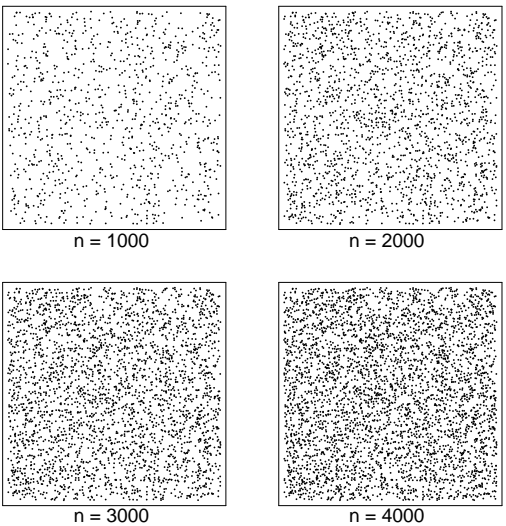

Figure 1. Comparison of sequences.

as possible in the error term (see e.g. [6]):

$$
\begin{aligned}
\mathbf{L}_{n} f-\mathbf{I} f & =\sum_{\vec{k} \in \mathbb{Z}^{d}} \tilde{f}(\vec{k}) \mathbf{L}_{n} \mathrm{e}^{2 \pi \mathrm{i} \vec{k} \cdot \vec{x}}-\tilde{f}(\overrightarrow{0}) \\
& =\sum_{\vec{k} \in L^{\perp}, \vec{k} \neq \overrightarrow{0}} \tilde{f}(\vec{k}),
\end{aligned}
$$

where $L^{\perp}=\left\{\vec{k} \in \mathbb{Z}^{d}: \vec{k} \cdot \vec{z}=0(\bmod n)\right\}$ is the reciprocal lattice.

\section{Implementation}

\subsection{Vegas}

Vegas is Lepage's classic Monte Carlo algorithm [7. It uses importance sampling for vari- 
ance reduction for which it iteratively builds up a piecewise constant weight function, represented on a rectangular grid. Each iteration consists of a sampling step followed by a refinement of the grid.

In CuBA's implementation Vegas can memorize its grid for subsequent invocations and it can save its internal state intermittently such that the calculation can be resumed e.g. after a crash.

\subsection{Suave}

Suave is a cross-breed of Vegas and Miser 8, a Monte Carlo algorithm which combines Vegasstyle importance sampling with globally adaptive subdivision.

The algorithm works as follows: Until the requested accuracy is reached, bisect the region with the largest error along the axis in which the fluctuations of the integrand are reduced most. Prorate the number of new samples in each half for its fluctuation.

The Vegas grid is kept across divisions, i.e. a region which is the result of $n-1$ subdivisions has had $n$ Vegas iterations performed on it. On the downside, Suave is somewhat memory hungry, as it needs to retain samples for later use.

\subsection{Divonne}

Divonne is a significantly extended version of CERNlib's Algorithm D151 9]. It is essentially a Monte Carlo algorithm but has cubature rules built in for comparison, too. Variance reduction is by stratified sampling, which is aided by methods from numerical optimization. Divonne has a three-phase algorithm:

\section{Phase 1: Partitioning}

The integration region is split into subregions of (approximately) equal spread $s$, defined as

$$
s(r)=\frac{\operatorname{Vol} r}{2}\left(\max _{\vec{x} \in r} f(\vec{x})-\min _{\vec{x} \in r} f(\vec{x})\right) .
$$

Minimum and maximum of each subregion are sought using methods from numerical optimization (essentially a quasi-Newton search). Then, 'dividers' are moved around (see picture) to find the optimal splitting. This latter procedure can cleverly be translated into the solution of a linear system and is hence quite fast (for details see [9]).

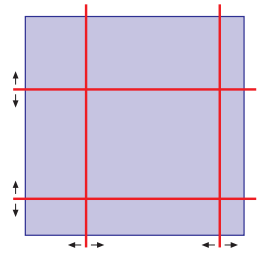

Phase 2: Sampling

The subregions determined in Phase 1 are independently sampled with the same number of points each. The latter is extrapolated from the results of Phase 1.

\section{Phase 3: Refinement}

Regions whose results from Phase 1 and 2 do not agree within their errors are subdivided or sampled again. This phase is an addition to the original algorithm since it was found that often enough the error estimate, or even the integral value, was off because characteristics of the integrand had not been found in Phase 1.

Two important features have been added in the CuBA implementation:

- The user can point out extrema for tricky integrands.

- For integrands which cannot be sampled too close to the border, a 'safety distance' can be prescribed within which values will be extrapolated from two points in the interior.

\subsection{Cuhre}

Cuhre is a deterministic algorithm. It uses the Genz-Malik cubature rules 2 in a globally adaptive subdivision scheme. The algorithm is thus: Until the requested accuracy is reached, bisect the region with the largest error along the axis with the largest fourth difference.

Cuhre has been re-implemented in CuBA mostly for a consistent interface, it is the same as the original DCUHRE subroutine [10].

\section{Comparison}

Doing a balanced comparison on integration algorithms is nearly hopeless. Performance depends highly on the integrand and there are always cases, and not just academic ones, in which one routine outperforms the others, or conversely, 
in which one routine simply gives wrong results. This, of course, is the main reason why there are four independent and easily interchangeable algorithms in the CuBA library.

In this context it should be pointed out that the statistical error estimate quoted by the Monte Carlo algorithms merely states the one-sigma interval, or in other words: the probability that the central value lies in the given interval is (only) about $68 \%$.

With these caveats, the following plot compares the performance of the four CUBA routines on a real phase-space integration. The results obtained by the four methods (not shown here) indeed agree to within the requested accuracy.

Integrand evaluations

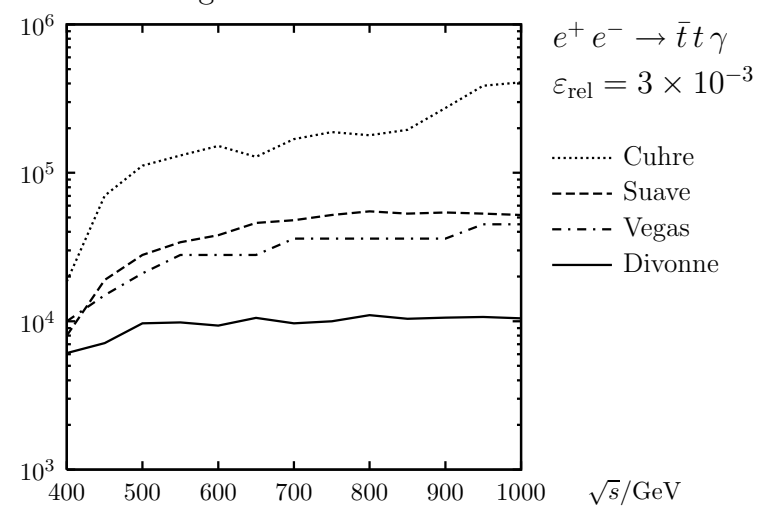

\section{Mathematica interface}

The Mathematica interface deserves a special mention, as it is not a library in the proper sense. It is rather four executables which communicate with Mathematica via the MathLink API:

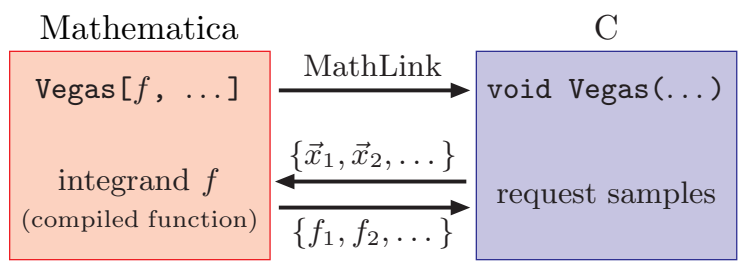

After loading the appropriate MathLink executable, e.g. with Install ["Vegas"], the corresponding routine can be used almost like Mathematica's native NIntegrate. The integrand is evaluated completely in Mathematica, which means that one can do things like
Cuhre $[$ Zeta $[\mathrm{x} y],\{\mathrm{x}, 2,3\},\{\mathrm{y}, 4,5\}]$

\section{Further Tools}

\subsection{Chooser}

CuBA includes a "one-stop interface" which further simplifies the invocation:

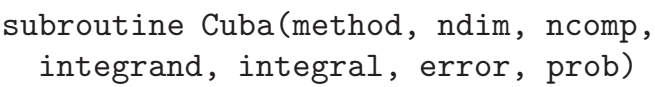

The user just has to choose method $=1,2,3,4$ to switch between Vegas, Suave, Divonne, Cuhre. All parameters specific to individual routines are "hidden" (determined inside the routine), i.e. this is not a finished product, but should be adapted by the user.

\subsection{Partition Viewer}

CuBA's Partition Viewer displays the partition taken by the integration algorithm. This is sometimes helpful to visualize where the integrand's characteristic regions lie. It is really useful only in small to moderate dimensions, though.

Verbosity level 3 must be chosen in the integration routine and the output piped through the partview utility, as in

myprogram | partview 12

which will then display the 1-2 plane of the partitioning. Figure 2 shows a screenshot.

\section{Summary}

CUBA is a library for multidimensional numerical integration written in C. It contains four independent algorithms: Vegas, Suave, Divonne, and Cuhre which have similar invocations and can be exchanged easily for comparison. All routines can integrate vector integrands and have a Fortran, $\mathrm{C} / \mathrm{C}++$, and Mathematica interface. Additional tools are included, such as a one-stop invocation and a partition viewer. CuBA is available at http://www.feynarts.de/cuba, licensed under the LGPL, and easy to build (autoconf).

\section{REFERENCES}

1. T. Hahn, Comp. Phys. Commun. 168 (2005) 78 hep-ph/0404043. 


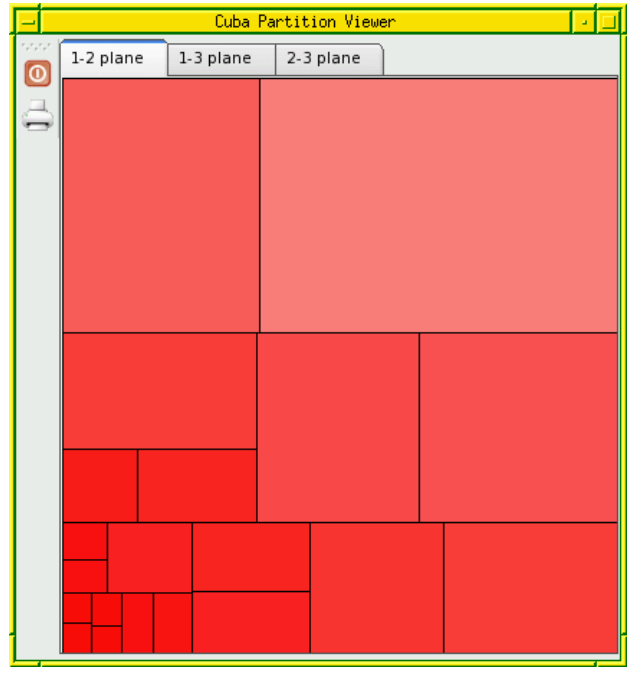

Figure 2. Screenshot of CUBA's partition viewer.

2. A. Genz, A. Malik, SIAM J. Numer. Anal. 20 (1983) 580.

3. W.H. Press, S.A. Teukolsky, W.T. Vetterling, B.P. Flannery, Numerical recipes in Fortran, $2^{\text {nd }}$ edition, Cambridge University Press, 1992.

4. P. Bratley, B.L. Fox, ACM Trans. Math. Software 14 (1988) 88. TOMS algorithm 659.

5. M. Matsumoto, T. Nishimura, ACM Trans. Modeling Comp. Simulation 8 (1998) 3 and http://www.math.sci.hiroshima-u.ac.jp/ $\sim \mathrm{m}$-mat/MT/emt.html.

6. H.L. Keng, W. Yuan, Applications of number theory to numerical analysis, Springer-Verlag, 1981.

7. G.P. Lepage, J. Comp. Phys. 27 (1978) 192. G.P. Lepage, Report CLNS-80/447, Cornell Univ., Ithaca, N.Y., 1980.

8. W.H. Press, G.R. Farrar, Comp. in Phys. 4 (1990) 190.

9. J.H. Friedman, M.H. Wright, ACM Trans. Math. Software 7 (1981) 76.

J.H. Friedman, M.H. Wright, SLAC Report CGTM-193-REV, CGTM-193, 1981.

10. J. Berntsen, T. Espelid, A. Genz, ACM Trans. Math. Software 17 (1991) 437 and 452. TOMS algorithm 698. 